\title{
Entretien avec Claire Hancock
}

\section{Claire Hancock}

\section{OpenEdition \\ Journals}

Édition électronique

URL : http://journals.openedition.org/rfst/453

DOI : $10.4000 /$ rfst.453

ISSN : 2492-3672

Éditeur

Espaces et SOciétés (UMR 6590)

\section{Référence électronique}

Claire Hancock, "Entretien avec Claire Hancock», Revue francophone sur la santé et les territoires [En

ligne], Genre, territoire et santé, mis en ligne le 21 décembre 2015, consulté le 06 avril 2021. URL http://journals.openedition.org/rfst/453 ; DOI : https://doi.org/10.4000/rfst.453

Ce document a été généré automatiquement le 6 avril 2021.

\section{(c) (i) (2) (2)}

La Revue francophone sur la santé et les territoires est mise à disposition selon les termes de la Licence Creative Commons Attribution - Pas d'Utilisation Commerciale - Partage dans les Mêmes Conditions 4.0 International. 


\title{
Entretien avec Claire Hancock
}

\author{
Claire Hancock
}

\section{NOTE DE L'ÉDITEUR}

Propos recueillis par Audrey Bochaton, Clélia Gasquet-Blanchard et Sandrine Halfen

\section{Biographie}

1 Claire Hancock est Professeure à l'Université Paris-Est Créteil et membre du LAB'URBA. Les travaux de Claire Hancock se situent dans le prolongement de courants géographiques postcoloniaux et féministes, avec pour thèmes centraux le genre et l'altérité, ainsi que les façons dont ils sont pensés et représentés spatialement. Claire Hancock aborde également dans ses recherches «l'habillage spatial du refus de l'Autre » ou l'instrumentalisation d'un vocabulaire de l'espace, ou du territoire, au service de discours d'exclusion des minorités.

\section{Le genre comme catégorie d'analyse en rapport avec les territoires}

\section{En quoi le genre apparaît-il comme une catégorie pertinente d'analyse de l'espace?}

2 Je ne pense pas le genre tant comme une "catégorie d'analyse" que comme un système de relations de pouvoir, au même titre que la classe ou la race par exemple; sachant que tous ces différents rapports de pouvoir s'articulent les uns aux autres, de manière complexe. C'est cette articulation que la recherche désigne sous le terme d'intersectionnalité. Tous ces rapports de pouvoir contribuent à la production de l'espace en même temps qu'ils sont produits et reproduits dans l'espace. Tel ou telle 
“n'est pas à sa place" dans tel ou tel espace : en ce sens, c'est l'espace qui fonctionne comme système de classement, de catégorisation des personnes.

\section{Quelles sont les positionnements scientifiques actuels sur le genre en sciences humaines, notamment en géographie et particulièrement concernant les questions de santé ?}

3 L'Institut Emilie du Châtelet a organisé un colloque, les 8 et 9 juin 2015, sur le croisement entre genre et santé, auquel je n'ai malheureusement pas pu assister... mais il est vrai que l'espace, ou la géographie, ne figuraient pas explicitement au programme de ce colloque! Ce qui est sans doute caractéristique de la difficulté à articuler ces trois champs, qu'on sait articuler deux à deux (genre et géographie, genre et santé, géographie et santé). Je pense qu'il y a sans doute un point d'entrée théorique dans la question par la thématique du care, du travail de soin, pris en charge massivement, dans nos sociétés comme dans beaucoup d'autres, par les femmes. Il me semble important de penser le care, la prise en charge du bien-être des autres (notamment enfants, personnes âgées, personnes en situation de handicap), non comme une disposition "naturelle" mais comme un travail à part entière, et comme un travail qui produit des pratiques et représentations spécifiques de l'espace. On ne perçoit pas l'espace urbain et ses équipements de la même façon lorsqu'on est assigné à des fonctions d'accompagnement, de prise en charge, et de soin; et pourtant ce sont des expériences spatiales, et des formes de travail, relativement négligées par une géographie androcentrée qui postule une partition entre espaces de vie et espaces de travail.

\section{Quelle acception avez vous du genre: en faites vous un champ d'étude à part entière ou bien un objet présent dans toute approche de géographie humaine?}

Le genre comme relation de pouvoir est bien sûr présent dans toute la géographie humaine (et physique, d'ailleurs! De jeunes collègues comme Anne Jégou, Edouard de Belizal et Antoine Chabrol l'ont bien montré), quand bien même celle-ci, dominée qu'elle a été historiquement par des perspectives androcentrées, l'ignore! Quant Michel Lussault écrit un livre intitulé "L'Homme spatial" (sic), il pense écrire depuis une perspective universelle, alors qu'il est évident qu'il ne décrit et ne réitère que des expériences construites à partir de sa propre position subjective masculine. Et c'est le cas de toute la géographie qui prétendant décrire le rapport entre "l'Homme et la Terre", reconduit une figure masculine du sujet humain, par opposition à une "mèrenature" féminisée... Donc toute la géographe baigne dans un discours genré qui s'ignore. Et ce n'est que la géographie dite "de genre", ou la géographie féministe, que je prône, qui prend en compte explicitement ce biais et tente de faire du genre un levier pour questionner des idées communément admises, et obliger à poser à nouveaux frais des questions à la géographie. 


\section{Comment le géographe peut aujourd'hui aborder les questions de genre?}

5 On constate que c'est souvent un processus difficile car se poser cette question, c'est accepter de pratiquer une réflexivité sur sa posture de chercheur, et s'interroger sur la position personnelle, subjective à partir de laquelle on produit du savoir, ce que Donna Haraway a appelé les "savoirs situés"; c'est aussi accepter de repenser qui on est et comment on s'est construit individuellement ou comment on a été construit par d'autres, dans une idéologie genrée. Pour beaucoup, il est plus simple de camper dans le déni, et de prétendre que cela n'a aucune importance ; c'est souvent la posture de ceux qui se pensent comme "objectifs" ou "neutres", sujets universels, parce que masculins, blancs, hétérosexuels, de classe moyenne ou aisée, valides... En revanche, c'est une remise en cause qui parle immédiatement, et qui fait sens, pour toutes celles et tous ceux qui sont ou ont été construits comme "spécifiques", "particuliers" par ce discours universalisant (femmes, minorités sexuelles, non-Blancs, etc). Pour aborder la dimension genrée des questions auxquelles on s'intéresse, il y a le niveau 0 , par lequel tout le monde peut entrer : introduire la variable homme/femme dans ses données, et voir ce qui en ressort. On découvre souvent des choses inattendues, mais qu'on ne sait pas forcément expliquer. On commence à "aborder les questions de genre", réellement, quand on commence à analyser et réfléchir à ce qui produit ces différences, ce qui suppose d'avoir recours aux apports de multiples disciplines, depuis la psychologie sociale jusqu'aux sciences politiques ou économiques; une approche genre est nécessairement pluridisciplinaire, ce qui oblige le ou la géographe à sortir de sa zone de confort... mais lui apporte aussi énormément!

\section{Articuler genre, territoire et santé}

\section{Existe-t-il des spécificités feminines et masculines en terme de représentations spatiales et de pratiques territoriales ? Comment ces différences peuvent-elles se traduire dans les comportements et les états de santé ?}

6 Je pense que plutôt que d'essentialiser et bi-catégoriser, il est utile d'emprunter à nos collègues scientifiques l'image du continuum (cf interview d'Anne Fausto-Sterling par Priscille Touraille ${ }^{2}$ ). Donc si je dis "les personnes qui prennent en charge le travail de care", je sais que ce seront en large part des femmes, et que cela conduit beaucoup de femmes à avoir cette expérience de l'espace, des réseaux de transport, des réseaux de soin, de l'accessibilité qui découle de leur travail de reproduction sociale. Mais cela n'en fait pas une expérience intrinsèquement "féminine", car parmi les personnes qui accomplissent ce travail, il se trouve aussi des pères isolés, des hommes prenant en charge des parents âgés, et des travailleurs étrangers, racialisés, qui sont également nombreux dans les secteurs du care... A l'inverse, une femme célibataire qui n'a pas la charge de personnes vulnérables ou en situation de handicap, ne partagera pas ces expériences. Donc plutôt que de penser masculin/féminin, il vaut mieux penser à des rôles sociaux, pris en charge préférentiellement par des personnes de sexe féminin (ou masculin), mais bien sûr pas exclusivement. Ce que montrent les études dont on dispose, c'est que ces responsabilités sociales font de certaines catégories de personnes 
(femmes, mais aussi personnes âgées, enfants, jeunes, personnes en situation de handicap, précaires...) des citadins plus "locally-dependent", plus tributaires des ressources du local, car moins mobiles, disposant de moins de ressources pour se déplacer. C'est pour ces personnes-là qu'il faudrait, en priorité, aménager, équiper, avec leurs besoins en tête; mais, malheureusement, même si ces personnes, toutes ensemble, représentent une majorité des citadins, elles sont sous-représentées parmi les personnes qui aménagent l'espace et prennent les décisions d'implantation des équipements. Ce n'est ni par elles, ni pour elles, qu'est produit l'espace, l'espace des équipements de santé comme les autres : ce sont bien au moins des inégalités, peut-être des injustices, qui président à ces aménagements. On tente parfois de nos jours de les atténuer par des démarches plus participatives, mais notre espace actuel est hérité de décennies, voire de siècles, où beaucoup d'usagers et d'usagères n'avaient pas voix au chapitre, étaient vu-e-s comme objet (du point de vue de la science biomédicale comme des producteurs de l'espace physique) et non comme acteurs et - trices à part entière (de leur santé comme de leur espace de vie).

\section{A votre avis, quel risque y a-t-il à spatialiser les questions de genre, particulièrement au regard des questions de santé qui peuvent dans certaines approches biomédicales participer à un déterminisme de genre?}

7 J'ai le sentiment, même si je ne connais qu'imparfaitement la littérature, qu'il y a une tendance à penser de manière essentialiste en santé, comme dans d'autres domaines d'ailleurs, en identifiant des pathologies "masculines" et "féminines", ce qui peut conduire, il y a eu des exemples récents dans la presse médicale, à sous-diagnostiquer les problèmes cardiaques chez les femmes, par exemple, ou à constater que les modèles de pacemaker qu'on produit le plus ne sont tout simplement pas adaptés aux morphologies féminines, d'où une surmortalité des femmes rencontrant ce type de problème! On est également effaré d'apprendre qu'une bonne partie des expérimentations biomédicales ont été historiquement conduites sur des populations animales uniquement masculines, et sans qu'on fasse attention au sexe des expérimentateurs, alors qu'on sait désormais que tous ces éléments biaisent et faussent les résultats de nombre d'études canoniques et considérées comme centrales dans différents domaines scientifiques. Et ne disons rien de la tendance à considérer que le genre, ça se réduit à la fonction reproductrice et maternante des femmes, et que c'est à ce titre qu'il faut prendre en compte les femmes...

\section{Vous travaillez à l'articulation entre les notions d'espace et de genre. Comment à votre avis peut-on articuler les notions de "Genre, Territoire \& Santé" ? Est-ce possible?}

8 Comme je le disais tout à l'heure, je crois que l'articulation des trois est complexe et délicate, qu'il faut à tout prix lutter contre les déterminismes simplistes : il me semble que se souvenir que ces trois notions sont des productions sociales est un point de départ essentiel, œuvrer à les dénaturaliser, et à mettre en évidence la trame de discours, d'images et de représentations qui les structure une étape importante. Peutêtre que l'image du continuum est une des plus utiles: on n'est dans aucun des trois cas 
en présence de faits qu'on pourrait délimiter sans ambiguïté et de manière binaire (homme/femme, dedans/dehors, santé/maladie), mais plutôt en présence de champs de bataille où se jouent beaucoup des tensions du social : des inégalités, des rapports de domination, des mobilisations... La posture féministe dont je me réclame consiste à la fois à insister sur l'agentivité des sujets (pas plus la patiente que la citadine ne doit être construite comme victime passive, elle est actrice de sa vie, de son espace de vie et de sa santé) et à insister sur le caractère profondément politique, et contestable, de situations qu'on voudrait nous faire prendre pour des fatalités ou des faits de nature.

\section{Pensez-vous que la construction des trajectoires sanitaires des populations peut être analysée à travers le genre?}

9 Je ne suis pas bien sûre de savoir ce que vous appelez "trajectoires sanitaires"... maintenant, si c'est quelque chose qui ressemble au "parcours de soins", il est évident que de multiples facteurs entrent en ligne de compte, dont l'accessibilité au sens large, c'est-à-dire définie non pas seulement en termes de moyens de transport ou de distance, mais aussi de dispositions sociales, culturelles, économiques... dont le genre. Mais on pourrait dire que les dispositions liées à la classe, et/ou la race, l'âge, la religion... sont autant de facteurs susceptibles d'infléchir le recours au soin, la façon dont on perçoit son corps, la souffrance, le volontarisme dont on peut faire preuve ou non, les ressources qu'on est en mesure de mobiliser (ressources économiques, mais aussi en termes de temps, de savoirs, de réseaux sociaux...). Il y a bien sûr des conditionnements sociaux également, comme celui d'un savoir médical masculin qui a construit la grossesse, par exemple, comme pathologie à "prendre en charge" et à médicaliser, alors que c'est une expérience somme toute commune! Il y a eu historiquement tendance à socialiser le corps des femmes, et à les déposséder de leur autonomie de décision par rapport à leur capacité reproductive : les Etats s'en mêlent, les médecins, les politiciens et les églises aussi, et on le voit autour des enjeux politiques que constituent la contraception, l'avortement, la GPA, la reproduction assistée... il serait évidemment illusoire de penser que le rapport des femmes à leur corps et à leur santé ressort indemne de tant de débats et de tant de politisation.

\section{Inégalités, justice sociale, genre et territoire : un engagement}

\section{L'observation d'inégalités de santé entre les hommes et les femmes relève-t-elle, outre de prédispositions biologiques, de constructions sociales et territoriales de genre?}

10 Je pense qu'il faudrait repartir de votre postulat même de "prédispositions biologiques": la plupart des phénomènes qu'on a longtemps considérés comme de l'ordre du naturel ou du biologique ont à force de recherche scientifique eu tendance à apparaître de plus en plus comme des productions sociales : prenez par exemple le fait qu'il naisse, en moyenne, 105 garçons pour 100 filles (le ratio est bien supérieur lorsqu'on discrimine à l'encontre de foetus féminins, dans certains pays d'Asie notamment). On a longtemps tenu un discours "voyez comme la nature est bien faite, parce qu'il y a surmortalité des jeunes garçons, la nature anticipe et en produit plus" - 
discours qui est de plus en plus remis en cause, car on découvre que plus les sociétés s'éloignent de la valorisation des enfants mâles, et plus le corps médical se féminise, plus cet écart s'amenuise, jusqu'à devenir insignifiant. Et il devient évident que par le passé, les médecins ont déployé plus de moyens pour sauver des fotus masculins, qu'ils valorisaient plus, de manière totalement inconsciente. De même, la surmortalité des jeunes garçons, qu'on lie souvent à leur plus grande vulnérabilité à certaines pathologies, est également produite par des sociétés qui ne découragent pas la prise de risques au même degré chez les garçons que chez les filles... Au-delà de ça, des travaux comme ceux de Anne Fausto-Sterling montrent bien, notamment à partir de la difficile question des tests de féminité dans le sport, que ce qui constitue une femme ou un homme est loin d'être une évidence biologique... je pense que comme pour la "race", dont plus personne ne soutient de nos jours qu'elle est une réalité biologique, on s'aperçoit que ce sont des rôles sociaux et des rapports de pouvoir qui produisent les individus, y compris dans leur identification à des traits physiques, et pas le contraire. On sait aussi que ces rapports sociaux produisent des pathologies et un mal-être spécifique des personnes qui ont à faire face à des discriminations et/ou qui sont cantonnées dans des rôles subalternes: ce que Christelle Hamel et d'autres ont montré pour des populations qui subissent le racisme en France ${ }^{3}$, notamment, et ce que des études états-uniennes ont aussi mis en évidence pour les femmes subissant le sexisme ${ }^{4}$. Ces personnes discriminées vont en outre subir plus d'expositions à des produits toxiques, être confinées dans des lieux de vie subissant plus de dégradations environnementales, et subir au quotidien des formes de violence et de déni de reconnaissance évidemment délétères pour leur santé. Mais encore une fois, en cela les incidences du genre sont compliquées par les appartenances de classe, race, le statut migratoire, l'âge, le statut d'emploi... On va donc constater des superpositions entre inégalités ou injustices spatiales, injustices environnementales (cadre de vie dégradé, sous-équipement médical, précisément là où les besoins des populations sont les plus importants) et des inégalités sociales généralement - mais sans qu'on puisse isoler le genre d'une multiplicité d'autres facteurs.

\section{Vous mobilisez fréquemment la notion de "justice spatiale" dans vos travaux, comment celle-ci peut constituer un apport intéressant aux géographes s'intéressant aux questions de santé ?}

11 Je pense, oui, et je le pense parce que différentes doctorantes en géographie de la santé de mon labo (Noémie Rapegno ou Mélina Ramos-Gorand) ont été amenées à mobiliser cette notion dans leurs travaux - ce n'est pas moi qui la leur suggère, mais je constate qu'elles s'en sont par elles-mêmes saisies, vraisemblablement parce qu'elle permet d'éclairer certaines situations pour lesquelles le terme d' "inégalités", tout seul, paraît inadéquat, insuffisant. Quand on en vient à porter dans son corps les stigmates ou les séquelles d'où on vit ou du rôle social subalterne qui nous est attribué, alors seul le terme d'injustice paraît assez fort: je pense par exemple à l'auteure états-unienne Linda Tirado qui a bien expliqué comment sa pauvreté est inscrite à tout jamais dans son visage faute de soins dentaires adéquats qu'elle ne pouvait se payer dans sa jeunesse... On pourrait penser aussi à ce que les Etats-uniens analysent en termes de "food deserts", la quasi-impossibilité, dans certains quartiers pauvres, de se procurer une nourriture fraîche et saine à des prix abordables, ce qui condamne une large part de la population à une alimentation peu équilibrée, malsaine, et donc à l'obésité et à 
toutes les pathologies liées aux carences ou au déséquilibre nutritionnel; ou plus près de nous, en Seine-Saint-Denis, au fait que la Caisse Primaire d'Assurance Maladie ferme des antennes dans le département métropolitain qui en a le besoin le plus aigu : on est bien là en présence d'injustices, qu'on gagne, je pense, à analyser dans leur dimension spatiale.

\section{A partir de vos écrits et vos thèmes de recherche, on présume un engagement}

12 Alors, bien sûr, et c'est ce qui gêne certains collègues dans l'utilisation des termes de justice ou injustice, cela veut dire qu'on sort d'une posture scientifique soi-disant neutre et "objective" pour prendre parti et énoncer un discours normatif : certains pensent qu'il faudrait s'en tenir à une description sans énoncer de jugement sur ce qui est juste ou non. Nous sortirions de la neutralité requise à la production d'un savoir scientifique... la réponse que je fais à ces critiques-là, c'est de dire qu'à mon sens, les discours soi-disant "neutres" sont en fait des discours complices du statu quo, et donc complices d'inégalités qu'ils se refusent à dénoncer. En cela ce sont des discours qui sont tout sauf objectifs : ne rien dire des rapports de pouvoir, c'est toujours prendre le parti des plus forts, qu'on le fasse consciemment ou non. On s'entend dire que la posture féministe ne serait pas scientifique: je pense, personnellement, qu'elle l'est bien plus que d'autres, parce qu'elle énonce clairement d'où elle parle, qui est un partipris et un engagement en faveur de l'égalité des femmes et des hommes. Pour moi tout discours qui ne se positionne pas explicitement comme posant cette égalité comme postulat de base est un discours susceptible d'être masculiniste, c'est-à-dire de nature à ignorer, entériner et/ou présenter comme naturelle une situation d'inégalité entre femmes et hommes.

\section{Pouvez-vous nous décrire les liens que vous percevez entre recherche, enseignement et engagement?}

13 C'est une articulation qui s'est faite pour moi progressivement, à partir d'un ancrage dans une université qui est une université dite "de banlieue", qui accueille un public "de banlieue" - Créteil, où j'ai fait mes premières armes d'enseignante il y a 20 ans maintenant. Après l'interlude de ma thèse en Sorbonne, j'y suis revenue en 1998 et j'ai développé progressivement une compréhension de ce contexte, et des jeunes qui en sont issus, qui m'a fait personnellement grandir et ouvrir les yeux sur tout un ensemble de rapports de domination que ma formation géographique des plus classiques, entre classes prépa et ENS, ne m'avait pas appris à voir et analyser. J'ai eu la chance de côtoyer ces jeunes qui m'ont appris beaucoup sur ce que c'est d'être l'Autre en France hors périphérique, hors institutions d'élite, altérisés, objectifiés, décrits depuis la perspective surplombante des médias et politiques dont ils et elles m'ont appris, progressivement, à me méfier. Donc si j'ai pu progresser en tant que chercheure, c'est vraiment grâce à ma pratique d'enseignante en milieu peu favorisé socialement : ces jeunes sont pour beaucoup intelligents et doués, mais découragés, rejetés par l'institution scolaire et la culture canonique. Me mettre à leur écoute est ce qui m'est arrivé de mieux. S'il y a un engagement de ma part, c'est, résolument, à leurs côtés. A côté de ça, je me suis beaucoup nourrie de la production géographique anglophone, et 
des travaux des féministes, qui affirment toutes deux, sur des modes différents, la nécessité de mettre sa pratique de chercheure en conformité avec ses convictions personnelles. Notre milieu universitaire est traversé d'inégalités et de rapports de pouvoir pour beaucoup genrés, il suffit de voir la composition des personnels administratifs, souvent des femmes, très mal reconnues et rémunérées alors que leur dévouement à l'institution est souvent considérable, qu'elles ne comptent ni leur temps, ni leur énergie... La souffrance au travail est considérable, surtout dans le contexte actuel du new public management appliqué à la hussarde. Il est important aussi d'ouvrir les yeux sur ces réalités du monde universitaire et de la recherche, et d'essayer de faire ce qu'on peut à son échelle, ou plus collectivement.

\section{Comment, à travers la thématique "Genre, Territoire \& Santé", ces liens peuvent-ils être tissés, alimentés, négociés ?}

"Santé", vous l'aurez compris, ça reste un univers de référence dont je peine, moi, à me saisir ; "genre", c'est un terme dont j'affectionne et je cultive le potentiel subversif, on comprend bien que s'il a gêné et suscité des réactions de la part des pans les plus conservateurs des la société, ce n'est pas pas hasard! Ce qui est plus navrant, c'est que des mouvements se prétendant de gauche se soient mis au diapason, et cultivent des visions parfois non moins navrantes... "Territoire”, enfin, est un mot vis-à-vis duquel je cultive la plus profonde méfiance, parce qu'il me semble qu'il a servi à occulter la dimension profondément idéologique de la construction d'identités territoriales, qui servent bien souvent à minimiser ou canaliser de réelles oppositions de classe, race, ou genre : c'est la construction artificielle de solidarités qui servent surtout les puissants, comme l'a montré Benedict Anderson avec le récit des identités nationales. Enfin, comme l'ont montré nombre de féministes depuis Virginia Woolf jusqu'à Rosi Braidotti, le discours du territoire est souvent un discours masculin, sinon viriliste, qu'il appartient aux féministes de contester et de déconstruire. A partir de là, peut-on construire quelque chose de suffisamment heuristique, puissant et transformateur autour de la thématique "genre, territoire et santé", c'est peut-être à vous de me le montrer?

\section{NOTES}

2. Anne Fausto-Sterling et Priscille Touraille, « Autour des critiques du concept de sexe. Entretien avec Anne Fausto-Sterling", Genre, sexualité \& société [En ligne], 12 | Automne 2014, mis en ligne le 01 décembre 2014, consulté le 29 juin 2015. URL : http:// gss.revues.org/3290 ; DOI : $10.4000 /$ gss.3290

3. https://www.ined.fr/fichier/s_rubrique/19579/ document_travail_2013_190_immigres_sante.fr.pdf

4. Laurel B. Watson, Jacob M. Marszalek, Franco Dispenza, Christopher M. Davids, "Under-standing the Relationships Among White and African American Women's 
Sexual Objectifica-tion Experiences, Physical Safety Anxiety, and Psychological Distress » Sex Roles, February 2015, Volume 72, Issue 3-4, pp 91-104

\section{RÉSUMÉS}

Cet entretien a été réalisé avec Claire Hancock, Professeure à l'Université Paris-Est Créteil et membre du LAB'URBA. Les travaux de Claire Hancock se situent dans le prolongement de courants géographiques postcoloniaux et féministes, avec pour thèmes centraux le genre et l'altérité, ainsi que les façons dont ils sont pensés et représentés spatialement.

Pour Claire Hancock, le genre ne se pense pas tant comme une "catégorie d'analyse" que comme un système de relations de pouvoir, au même titre que la classe ou la race par exemple. Elle se réfère à la notion d'intersectionnalité pour désigner l'articulation complexe de ces différents rapports de pouvoir combinés les uns aux autres. Selon elle, tous ces rapports de pouvoir contribuent à la production de l'espace en même temps qu'ils sont produits et reproduits dans l'espace. Quand nous avons transmis à Claire Hancock notre questionnement sur la manière d'articuler Genre, Territoire et Santé, elle nous a révélé trouver l'articulation de ces trois termes à la fois complexe et délicate car présentant le risque de tomber dans des déterminismes simplistes. Pour éviter cet écueil, elle suggère de garder à l'esprit que ces trois notions sont des productions sociales et non des faits qu'on pourrait délimiter sans ambiguïté et de manière binaire (homme/femme, dedans/dehors, santé/maladie). Par l'intermédiaire de notions telles que celle du continuum ou encore de justice spatiale, Claire Hancock offre des pistes riches et engagées pour porter plus loin notre réflexion.

This interview was conducted with Claire Hancock, Professor of geography at the University Paris-Est Creteil and member of LAB'URBA. Her research is in the continuation of postcolonial and feminist geographies and her main themes include gender and otherness, and the ways they are reflected and spatially represented.

For Claire Hancock, gender should not be thought of as a category for analysis but rather as a system of power relations, as well as class or race. She introduces the concept of intersectionality to name the complex articulation between these different power relationships combined with one another. According to her, all of these power relationships contribute to the production of the space and, at the same time they are produced and reproduced within the space.

For Claire Hancock, in order to address the gendered perspective of a research question, there are two levels. First, there is the level 0 that everyone can complete by introducing the feature man/woman in its data and see what findings come out. Through this, we often discover unexpected things, but we do not necessarily know to explain it. However, Claire Hancock asserts that we really start to address gender issues when we start to analyze and think about the factors that produce these differences between women and men. This requires to use inputs and perspectives from different fields including, social psychology, political sciences or economy. For her, a gendered approach is necessarily multidisciplinary and requires that the geographer steps out of its comfort zone.

About our questioning on the way to bring together the notions of Gender, Space and Health, Claire Hancock admits that she finds this articulation both complex and sensitive as it presents the risk to fall into simplistic determinism. To tackle this problem, she suggests keeping in mind that these three notions are social productions and not facts that we can delineate distinctly and 
in a binary way (man/woman, inside/outside, health/disease). Through concepts such as "continuum" or "spatial justice", Claire Hancock provides rich and committed points for further reflection.

\section{AUTEUR}

\section{CLAIRE HANCOCK}

Professeure à l'Université Paris-Est Créteil et membre du LAB'URBA 